\title{
Computer Simulation of Final-Stage Sintering: I, Model, Kinetics, and Microstructure
}

\author{
Gregory N. Hassold ** I-Wei Chen * and David J. Srolovitz \\ Department of Materials Science and Engineering, University of Michigan, \\ Ann Arbor, Michigan $\quad 48109-2136$
}

\begin{abstract}
A Monte Carlo model for simulating final-stage sintering has been developed. This model incorporates realistic microstructural features (grains and pores), variable surface diffusivity, grain-boundary diffusivity, and grain-boundary mobility. A preliminary study of a periodic array of pores has shown that the simulation procedure accurately reproduces theoretically predicted sintering kinetics under the restricted set of assumptions. Studies on more realistic final-stage sintering microstructure show that the evolution observed in the simulation closely resembles microstructures of real sintered materials over a wide range of diffusivity, initial porosity, and initial pore sizes. Pore shrinkage, grain growth, pore breakaway, and reattachment have all been observed. The porosity decreases monotonically with sintering time and scales with the initial porosity and diffusivity along the grain boundary. Deviations from equilibrium pore shapes under slow surface diffusion or fast grain-boundary diffusion conditions yield slower than expected sintering rates. [Key words: sintering, computers, theory, grain growth, microstructure.]
\end{abstract}

\section{Introduction}

$\mathbf{F}$ INAL-STAGE sintering is distinguished from earlier stages of sintering by two characteristics. First, no continuous channel of pore space exists in the aggregate. ${ }^{1}$ Individual pores are either of a lenticular shape (if they reside on the grain boundaries) or are rounded (if they are within a grain). The second major signature of the onset of late-stage sintering is an increase in the pore and grain-boundary mobilities. ${ }^{2-5}$ Pore migration, pore coalescence, pore breakaway (from grain boundaries), and grain growth all become important in finalstage sintering. The above two characteristics are presumably interrelated. Indeed, if a continuous channel of pore space spans the junction lines along which three grains meet, neither the pore channel nor the grain boundaries are mobile.

Final-stage sintering kinetics resemble that of an exhaustion process, proceeding at an ever-decreasing rate toward the end state. ${ }^{1}$ The end point density of a sintered body, however, is frequently dictated by the amount of residual porosity trapped within the grains as a result of pore breakaway from migrating boundaries during grain growth. ${ }^{2}$ These observations strongly suggest that, although surface and lattice diffusion may be important in earlier stages of sintering, it is

T. Shaw-contributing editor

Manuscript No. 197912. Received December 15, 1989; approved June 14 , 1990.

Presented at the 91st Annual Meeting of the American Ceramic Society, Indianapolis, IN, April 25, 1989 (Basic Science Division, Paper No. 62-B-89). Supported by the University of Michigan (GNH and DJS) and the U.S. Department of Energy (BES), Grant No. DE-FG02-87ER 45302 (IWC).

* Member, American Ceramic Society.

'Present address: Department of Science and Mathematics, GMI Engineering and Management Institute, Flint, MI, 48504. grain-boundary diffusion that is primarily responsible for pore shrinkage in final-stage sintering. Nevertheless, surface diffusion may be pertinent for pore mobility, ${ }^{6,7}$ and thereby it may affect both microstructure and sinterability. ${ }^{4,5}$

Even if pores do not break away from grain boundaries, so that the theoretical density is eventually achieved, no universal kinetics for pore shrinkage, to our knowledge, has been established. This uncertainty in sintering kinetics probably reflects the microstructural, microphysical, and microchemical variability inherent in the initial state (especially if they affect the path of final-stage sintering). Examples of such variability include, but are not limited to, grain-boundary and surface energies and diffusivities, grain-size and pore-size distributions, residual stresses, and chemical composition. Existing models of final-stage sintering. ${ }^{1,3-5,8-10}$ have mostly ignored such complications and have dealt with simplified, hypothetical (usually periodic) configurations of pores and grain boundaries. In many respects, such models have failed to quantitatively predict the kinetics and microstructures of final-stage sintering. Clearly, an alternative approach to the sintering problems which accounts for the above aspects of final-stage sintering would be desirable.

In this and the following paper ${ }^{11}$ (hereafter referred to as part II), we report a new approach to studying late-stage sintering which employs a hybrid Monte Carlo computersimulation algorithm. This approach is built upon a methodology that was previously developed to simulate grain growth, ${ }^{12,13}$ recrystallization, ${ }^{14,15}$ and other forms of microstructural evolution. ${ }^{16,17}$ Using this technique, we are able to treat microstructural complexity by including grain-boundry and pore topology, as well as their detailed environments, and sample a large statistical ensemble of such configurations. Multiple kinetic mechanisms are incorporated by assigning different Monte Carlo probabilities to various rate processes corresponding with the large variety of experimental conditions encountered in sintering. Although our present simulations have been performed in two dimensions, the resultant microstructures may be compared with cross sections of true threedimensional materials.

The present paper begins by examining the details of the model and the simulation techniques. A simulation of the evolution of a bicrystal containing periodically spaced pores along its grain boundary is then used to calibrate the pore-shrinkage kinetics and is followed by simulations of more realistic microstructures of grains and pores. In presenting and discussing these results, emphasis will be placed on the role of the basic atomic processes. Part II ${ }^{11}$ analyzes the grain and pore topology, their size distributions and correlations, the sintering $\mathrm{ki}$ netics, and the scaling laws governing the microstructural correlations and evolution during late-stage sintering.

\section{Model and Simulation Method}

\section{(I) Mapping of Grains and Pores}

To incorporate the shape and size distributions of grains and pores into our simulation, the microstructure is mapped onto a discrete triangular lattice (Fig. 1). The basic technique 


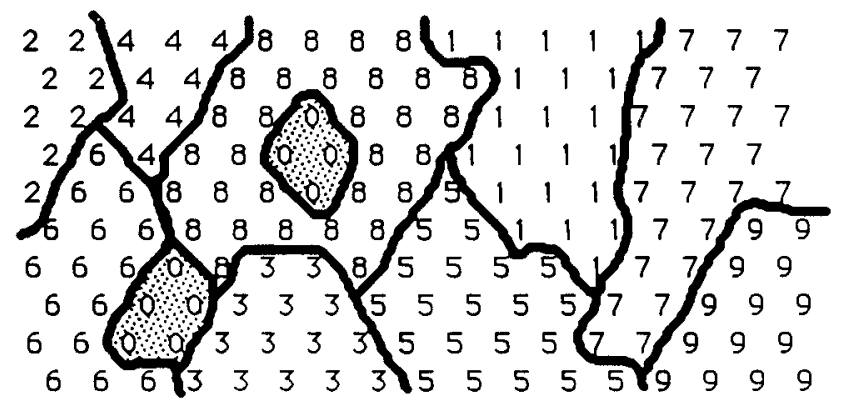

Fig. 1. Mapping of a microstructure onto a triangular lattice. Solid lines represent grain boundaries or surfaces, positive numbers at each site indicate the local orientation of the grain in the vicinity of the site, and zeroes and shading indicate pores.

is identical with the one used by Anderson et al. ${ }^{12}$ in their computer simulations of grain growth, with a modification to account for pores. Each lattice site is assigned a number between 1 and $Q$, corresponding to the orientation of the grain in which it is embedded. In the case of pores, the lattice site is assigned a number zero. In analogy with a magnetic system, we will refer to these site-orientation numbers as spins. A sufficiently large number of possible grain orientations $(Q)$ are chosen so that grains of like orientations impinge infrequently $(Q=100)$. In our previous studies on grain growth and other phenomenon, ${ }^{12-17}$ we have already confirmed that the simulation results of different $Q$ are nearly indistinguishable from each other when $Q>50$ is used.

\section{(2) Energetics of Grain Boundaries and Pore Surfaces}

The grain-boundary and surface energies $\left(E_{g b}\right.$ and $E_{s}$, respectively) are specified by defining interactions between nearest-neighbor lattice sites. The Hamiltonian describing these interactions is

$$
H=\sum_{n n}\left(1-\delta_{S_{i} S_{j}}\right) J\left(S_{i} S_{j}\right)
$$

where $S_{i}$ is the spin $0 \leq S_{i} \leq Q, \delta_{a b}$ is the Kronecker delta, $J(x)=E_{g b}$ if $x>0$, and $J(x)=E_{s}$ if $x=0$. The sum is taken over all nearest-neighbor ( $n n)$ sites. Thus, nearest-neighbor pairs contribute $E_{g b}$ to the system energy when they are of unlike orientation, i.e., at the grain boundary. They contribute $E_{s}$ to the system when only one of the pair is zero, i.e., at the pore surface. Neighboring pairs of like orientation are within the same grain and neighboring pairs of zeroes are within the same pore; this establishes the zero of the energy scale. In this way, the excess energy at $0 \mathrm{~K}$ may be fully attributed to grain boundaries and free surfaces. The reference free energy of the bulk, of both the grains (solid) and the pores (vacuum), is taken to be zero.

Note that both the grain-boundary and surface energies have a slight anisotropy because of the mapping of the model onto a lattice. The orientation dependence of the energy of a straight interface segment exhibits a ratio of the maximum to minimum interfacial energy of $2 / \sqrt{3} \approx 1.15$ on the triangular lattice. Because this ratio is close to unity, both interfacial energies may be viewed as being nearly isotropic.

\section{(3) Kinetics}

(A) Boundary Motion: The kinetics of boundary migration are simulated by employing a Monte Carlo technique. ${ }^{12}$ In this method, a lattice site is chosen at random; if it is a grain site $\left(S_{i}>0\right)$, a new trial orientation from 1 to $Q$ is randomly selected. If the new trial configuration lowers the total energy of the system, according to Eq. (1), the new orientation is accepted; otherwise, the old value is retained. Such a sequence of steps has the effect of lowering the total grainboundary energy during the course of microstructural evolu- tion. Indeed, it simulates the process of normal grain growth as demonstrated previously by Anderson et al. ${ }^{12,13}$ Because such a reorientation will occur only when the site is on the grain boundary and the new orientation is that of the adjacent grain (except at the earliest times), this process may be viewed as an "atomic" jump across the grain boundary, with a net result of causing grain-boundary migration. Later, we will refer to the rate at which this "diffusion" or migration process occurs as $D_{m}$.

(B) Surface Diffusion of "Vacancies": If the randomly chosen site at any step of the simulation belongs to a pore, then one of its neighboring sites is selected, at random, and a trial exchange of the two spins between these sites is attempted. The energy change $\Delta E$ caused by the exchange is computed according to Eq. (1). Although such exchanges could be accepted only when $\Delta E \leq 0$, spin-exchange dynamics are more susceptible to the lattice anisotropy than the spin flip (reorientation) dynamics used for boundary migration. As a consequence, the exchange is performed with a probability

$$
P=q[1-\tanh (\Delta E / 2 k T)]
$$

where $T$ is a fictitious temperature associated with this process. This finite temperature transition probability ameliorates some of the effects of the anisotropy/discreteness of the lattice. Note that, except for a factor $q$, the probability is essentially zero if $\Delta E>>k T$, and is unity if $\Delta E<<0$. The prefactor $q(<1)$ dictates that such transition occurs only occasionally.

Although the algorithm previously described permits surface diffusion at any rate slower than $D_{m}$, in reality, surface diffusion may actually occur faster than the atomic jumps across the grain boundary which lead to grain-boundary migration. This limitation can be removed by employing a bookkeeping algorithm that distinguishes grain-boundary sites from surface sites and allowing updates of the surface sites more frequently in the Monte Carlo sampling (i.e., $q>1$ ). In this way, the rate of surface diffusion can be varied without restriction. We will refer to the rate at which these surface diffusion attempts occur (implemented by the above sampling algorithm described earlier and Eq. (2)) as $D_{s}$.

(C) Pore Shrinkage: In the present simulation, we will assume that pore shrinkage occurs by grain-boundary diffusion only. To envision this process, we may select a pore at random and allow it to shrink, with a certain probability, by removing a vacancy at one of its junctions with the grain boundary. Physically, pore shrinkage involves outward diffusion of the vacancies along grain boundaries and shedding them uniformly between pores along the grain boundary. ${ }^{18}$ Furthermore, the driving force for pore shrinkage comes from the pore surface tension and, hence, is inversely proportional to the pore size $r$. In two dimensions, the diffusion distance for this vacancy diffusion is of the order of the pore spacing $\lambda$. Therefore, we may write the shrinkage probability as

$$
\mathscr{P}=\frac{D}{r \lambda}
$$

where $D$ is a scaling constant. A rigorous proof of Eq. (3) is given in Appendix A for periodically spaced pores along a grain boundary.

To implement this model in our simulation, we note that both $r$ and $\lambda$ vary from pore to pore. In particular, the pore spacing pertaining to the diffusion distance of vacancies emitted from a pore cannot be easily estimated. Indeed, a diffusing vacancy will execute a random walk along the grainboundary network, as shown in Fig. 2, until it reaches another pore. The mean-squared distance a random walker gets from its origin is proportional to the number of steps it has taken, $N$.

Motivated by these considerations, we may identify $\lambda$, as a root-mean-square distance (proportional to $N^{1 / 2}$ ), and rewrite 


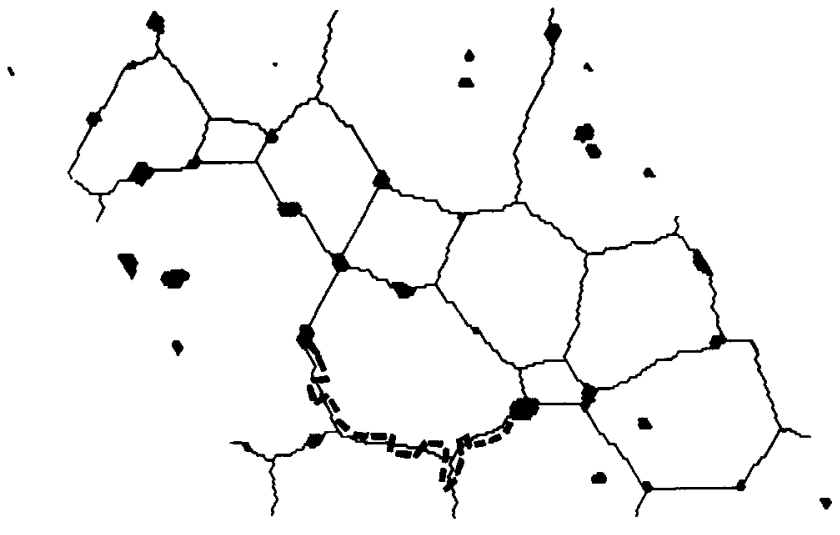

Fig. 2. Random walk of a diffusing vacancy along a grainboundary network. Solid lines indicate grain boundaries, dark regions indicate pores, and dashed line indicates a possible random walk path.

Eq. (3) as follows:

$$
N=\left(\frac{D}{r \mathscr{P}}\right)^{2}
$$

When a vacancy site at the junction of a grain boundary with a pore is chosen during the random sampling, then it will be allowed to wander along the grain-boundary path for $N$ steps in any direction. If it has reached another pore by the end of the $N$ steps, the initial site is reassigned to become a grain site with an orientation matching either one of the adjoining grains, and the vacancy is removed from the system. (The latter process is equivalent to placing the vacancy at the outer surface of the simulated body, i.e., shrinkage on a macroscopic scale.) If the random walk fails to reach another pore, then the initial configuration remains and the simulation proceeds. In Eq. (4), $r$ is calculated by counting the total number of sites in the pore and taking its square root. In this way, we are able to implement the basic kinetic law, dictated by Eq. (3), while giving a proper account to the variations of $r$ and $\lambda$ in the local microstructure. The essence of diffusion which governs pore shrinkage is embodied by the randomwalk process.

Finally, in order that the shrinkage lowers the total energy, the energy change in converting to the final configuration is computed and the entire process is accepted with a probability given by $[1-\tanh (\Delta E / 2 k T)]$. (This is essentially equivalent to the kinetics dictated by the absolute rate theory.) The rate at which the pore shrinkage occurs is determined by the value of $D / \mathscr{P}$, which thereby sets the rate of atomic diffusivity along the grain boundary necessary for pore shrinkage. Later, we will refer to the rate at which this process proceeds as $D_{g b}$.

\section{(4) Time Scale, Size Scale, and Boundary Conditions}

The time scale used in the simulation is designated as that required to choose all of the lattice sites once for an attempted change, whether it is successful or not. We refer to this time as one Monte Carlo step (MCS). Because the present simulations are performed on $200 \times 200$ lattices, 1 MCS corresponds to $10^{4}$ attempts. Typical simulation runs last from $10^{4}$ to $10^{7} \mathrm{MCS}$, depending on the choice of parameters. These systems sizes and times are at least comparable with the ones we used previously for successful simulations of grain growth and other similar phenomenon. ${ }^{12-17}$ They are deemed sufficient to avoid artifacts.

Although we have associated lattice sites in our simulation with vacancies and atoms, the artificial lattice created here is mainly for the convenience of designating microstructural characteristics and not for representing atomistics. Thus, the growth or shrinkage of a grain or a pore by one site would be more appropriately interpreted as an evolution increment by a unit volume. Indeed, we have used macroscopic rate equations only in directing the simulation process performed on each site. It is in this sense that we simulate grain-boundary migration $\left(D_{m}\right)$, surface diffusion $\left(D_{s}\right)$, and pore shrinkage through grain-boundary diffusion $\left(D_{g b}\right)$.

Periodic boundary conditions are imposed in both directions in all simulations. In all, five independent trials have been averaged over for each set of parameters to obtain data without undue statistical fluctuations.

\section{Sintering of a Periodic Array of Pores}

To check the validity of our method, the sintering of a periodic, linear array of pores along a grain boundary was simulated and compared with the analytical theory underlying Eq. (3). The initial size, shape, and spacing of all the pores were identical. To ascertain the effect of various parameters that enter the shrinkage equation, we varied the initial pore spacing (by a factor of 2) and the ratio of $D_{g b}$ to $D_{s}$ (from 0.1 to 1.0$)$. The ratio of surface to grain-boundary energy, $E_{s} / E_{g b}$, was set equal to unity, in agreement with data on typical oxide ceramics. ${ }^{19}$ The thermal energy $k T$ was set at $0.5 k T_{m}$, where $T_{m}$ is the temperature at which the present spin model "melts" or disorders. The surface diffusivity was chosen such that pore equilibrium proceeds much faster than grain growth, i.e., $D_{s} / D_{m}=10$.

Figure 3 shows a series of micrographs taken for a simulation with an initial pore-separation $\lambda$ (center to center) of 45 (lattice constants) and a pore radius of 5 (lattice constants). The pores are initially hexagon shaped, which is the equilibrium shape in the triangular lattice when $E_{s}=E_{g b}$. As sintering proceeds, the pores shrink and become less regular but are still compact and relatively equiaxed, because the dihedral angle is $120^{\circ}$ when $E_{s}=E_{g b}$. The nonuniformity in grain shape observed is a result of both the discreteness of the lattice and the finite temperature surface diffusion kinetics. Note that the grain boundary remains straight between pores but moves so as to connect the pores at their points of minimal separation. This is expected from the minimization of
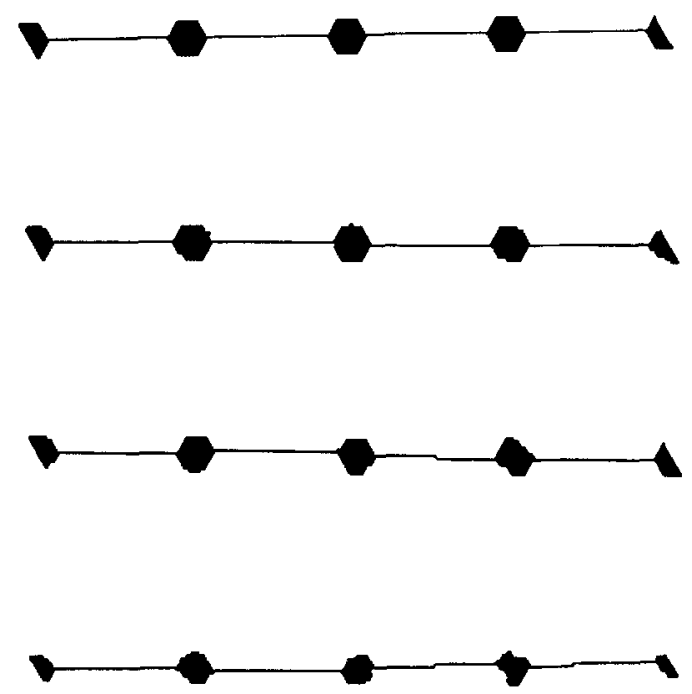

Fig. 3. Time evolution of a periodic array of pores along a straight grain boundary. Microstructures correspond to (top to bottom) times of $0,10^{4}, 3 \times 10^{4}, 10^{5}$, and $3 \times 10^{5} \mathrm{MCS}$. 
the grain-boundary energy. Similar observations have been made in other simulations at different initial pore sizes and pore spacing.

The kinetics of the pore shrinkage are summarized in Fig. 4. According to the theoretical analysis in Appendix A, the pore size, in a periodic array, should decrease with time as

$$
r^{3}=r(0)^{3}-\frac{C D_{g b} t}{\lambda}
$$

where $C$ is a constant and $r(0)$ is the initial pore size. To make a direct comparison with this prediction, the data in Fig. 4 are plotted by normalizing pore size by the pore spacing $(\lambda)$ and time $\lambda^{4} / D_{g b}$. The data, normalized in this manner, exhibit a slope of unity in Fig. 4; furthermore, they cluster together along the same straight line, as expected from Eq. (5). Note that the data for higher $D_{g b}$ suggest that the microstructures sinter slightly more slowly (in these normalized units) compared with rest of the data. This is possibly due to the less complete equilibration of the pore shapes during densification. In all, the agreement among the different data sets is good, and the theoretically predicted linear relationship appears to hold over at least 4 orders of magnitude.

\section{Microstructure Evolution and Kinetics During Final-Stage Sintering}

Figure 5 shows the temporal evolution of a typical polycrystalline microstructure during final-stage sintering. The initial microstructure was prepared to a specified initial porosity and pore size by performing a grain-growth simulation until the grain size reached the desired pore size, then randomly reassigning grains as pores until the desired porosity was achieved. (The procedure for simulating grain growth follows that reported by Anderson et al. ${ }^{12}$ ) The preparatory simulation was then continued until further growth was arrested by the pinning of grain boundaries on the pores. During the latter step, no sintering was allowed, i.e., $D_{g b}=0$ but $D_{m} \neq 0$. The initial porosity was chosen to be on the order of $9 \%$, which was appropriate for the onset of final-stage sintering without connected pore space (Appendix B). ${ }^{1}$ The initial (linear) pore and grain sizes were 3.5 and 15.7 , respectively.

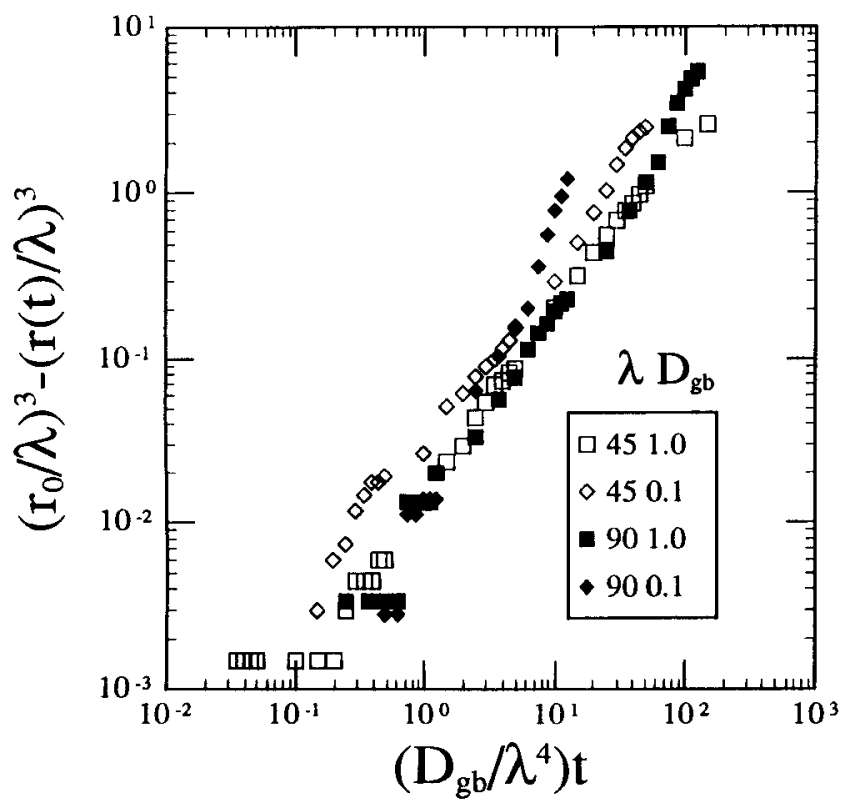

Fig. 4. Normalized pore shrinkage kinetics for regular arrays of pores for simulations using different pore spacings $\lambda$ and grainboundary diffusivity $D_{g b}$.

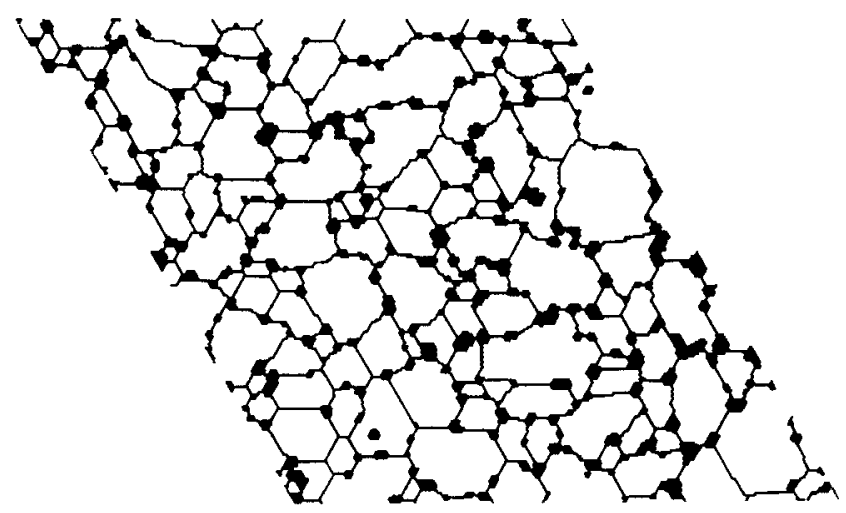

(A)

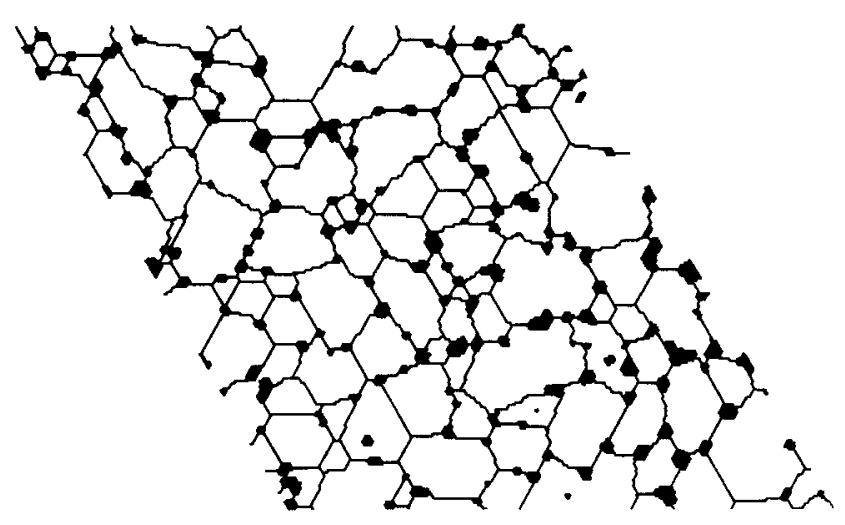

(B)

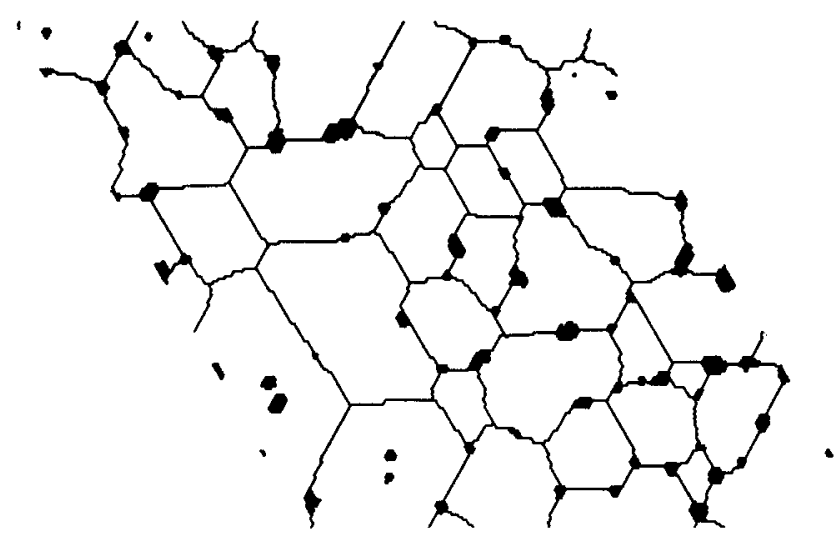

(C)

Fig. 5. Microstructural evolution of a porous polycrystalline solid undergoing final-stage sintering where $D_{\text {gb }} / D_{m}=0.1$ and $D_{s} / D_{m}=$ 1: (A) 1 , (B) $3 \times 10^{4}$, and (C) $3 \times 10^{5}$ MCS. Initial porosity, pore size, and grain size were $9.8 \%, 3.5$, and 15.7 , respectively.

All other parameters were set to their standard values, i.e., those noted in the previous section.

Several salient features may be noted in Fig. 5. Densification is clearly evident in these micrographs. Pores that are either larger than the mean pore size or relatively isolated appear to shrink more slowly than the mean pore size. Significant grain growth is seen only after the pores begin to disappear. Depinning of a grain boundary may occur either by the shrinkage of a pore, by a grain boundary pulling off of a pore, or by some combination of these. When a grain boundary pulls away from a pore, the pore remains within a grain and does not shrink until, at a later time, it is reattached to a moving grain boundary that happens to come its way. Pores along grain boundaries equilibrate toward a lenticular 
shape, although the discreteness of the triangular lattice and the diffusion of inidividual vacancies precludes the attainment of ideal, smooth shapes. Careful comparison of the microstructures at different times also reveals the cooperative motion of pores and grain boundaries so as to minimize the grain-boundary energy, although at a rate much slower than that of normal grain growth.

The decrease of porosity with sintering time is shown in Fig. 6. Included in this plot are data from three sets of simulations with different initial porosity. On this plot (porosity, $\Phi$, versus $\log t$ ), these data show a characteristic sigmoidal shape. This can be seen more readily in the normalized plot, Fig. 7, in which $\Phi$ is divided by the initial porosity, $\Phi_{0}$. The initial flat portion of the sintering curve simply reflects the very short initial time period which is insufficient for any sintering action to occur. The final flat portion is mostly due to the residual porosity trapped within the grains, which may be removed only when/if the pores become reattached to the grain boundaries after subsequent grain growth. The very long time required for the latter process suggests that this reattachment process may not be observable in typical sintering experiments, which last only for hours. Thus the shrinkage rate, $\mathrm{d} \Phi / \mathrm{d} t$, is a decreasing function of time once the initial "incubation period" has passed, in agreement with common experimental observations that sintering kinetics resemble that of an exhaustion process. ${ }^{1,20}$

To further investigate the role played by grain-boundary diffusion in the sintering process, we have performed a set of simulations in which the value of $D_{g b} / D_{s}$ is varied from 0.01 to 10.0. These results are displayed in Fig. 8, which shows that the sintering rate slows with decreasing $D_{g b} / D_{s}$. After normalization of the sintering time by $D_{g b}$ (see Fig. 9), all the data sets collapse onto a single curve with only small deviations. However, it is obvious that the simulations with higher $D_{g b}$ values sinter slower (in normalized time), as shown in the simulations of the periodic array of pores. A closer examination of the microstructures from the simulation with the highest grain-boundary diffusivity (Fig. 10), reveals that the pores are less lenticular compared with the ones in Fig. 5 (where $D_{g b} / D_{s}=0.01$ ), fewer grain boundaries appear to be pinned by pores, and both the grain size and porosity appear to be larger. Thus, allowing pore shrinkage to proceed too rapidly apparently hampers the attainment of full equilibration of the pore shape and decreases their effectiveness as pinning obstacles for grain-boundary motion. As a lenticular-

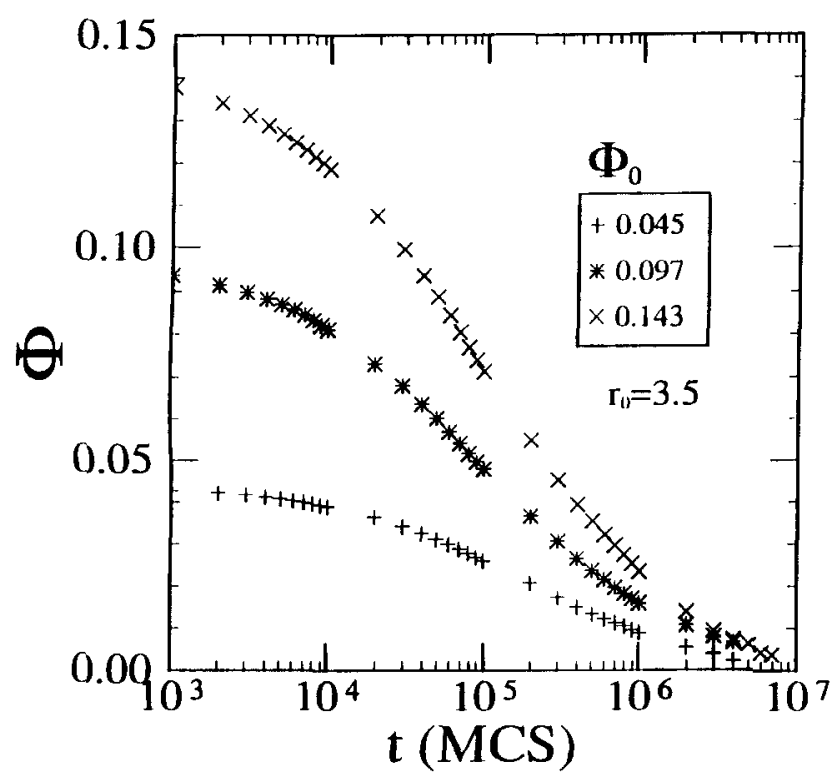

Fig. 6. Dependence of the porosity evolution kinetics on the initial porosity $\Phi_{0}$ where $D_{g b} / D_{m}=0.1$ and $D_{s} / D_{m}=1$.

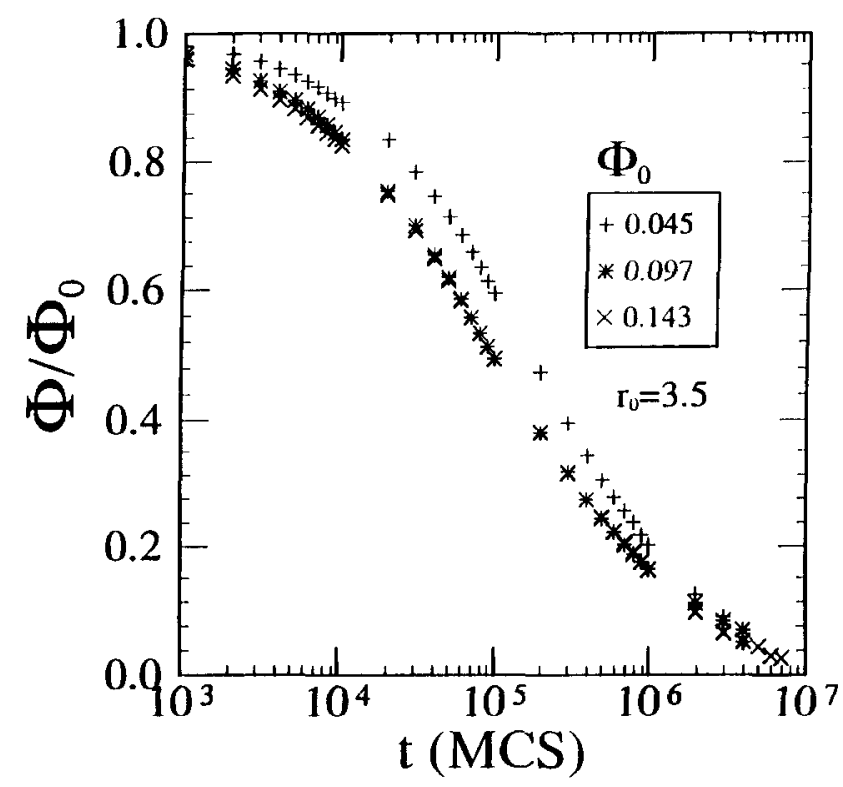

Fig. 7. Dependence of the porosity evolution kinetics on the initial porosity $\Phi_{0}$, where $D_{g b} / D_{m}=0.1$ and $D_{s} / D_{m}=1$. Porosity has been normalized by the initial porosity $\Phi_{0}$.

shaped pore intersects a larger section of a grain boundary than does an equiaxed pore (for dihedral angles less than $180^{\circ}$ ), it is easier for a grain boundary to pull off of a nonequilibrated pore than an equilibrated one.

\section{Discussion}

We have introduced three quantities into the present sintering model which govern the rates of the elementary kinetic processes during final-stage sintering. These are as follows: the surface diffusivity $\left(D_{s}\right)$, pertaining to the site exchange of a vacancy on the pore surface; the grain-boundary diffusivity $\left(D_{g b}\right)$, pertaining to the pore shrinkage via Eq. (3); and another rate constant $\left(D_{m}\right)$, pertaining to the site exchange of an atom across the grain boundary and, hence, the grainboundary mobility. Lattice diffusivity is not introduced into the present model. Note that, in a pure substance, $D_{g b}$ and $D_{m}$

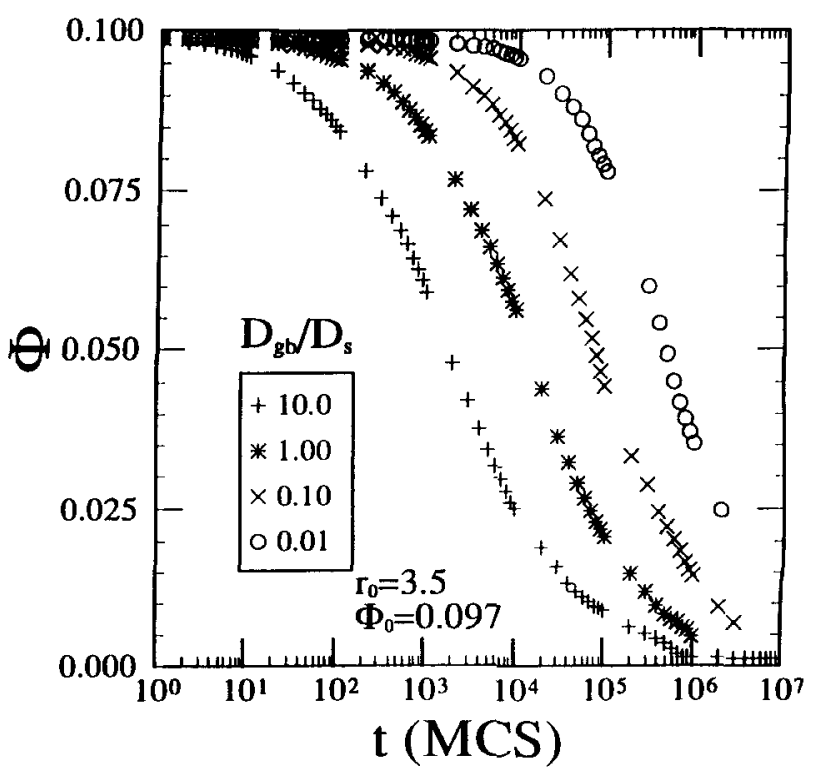

Fig. 8. Dependence of the porosity evolution kinetics on the ratio of $D_{g b} / D_{s}$, where the initial porosity, pore size, and grain size were $9.7 \%, 3.5$, and 15.7 , respectively. 


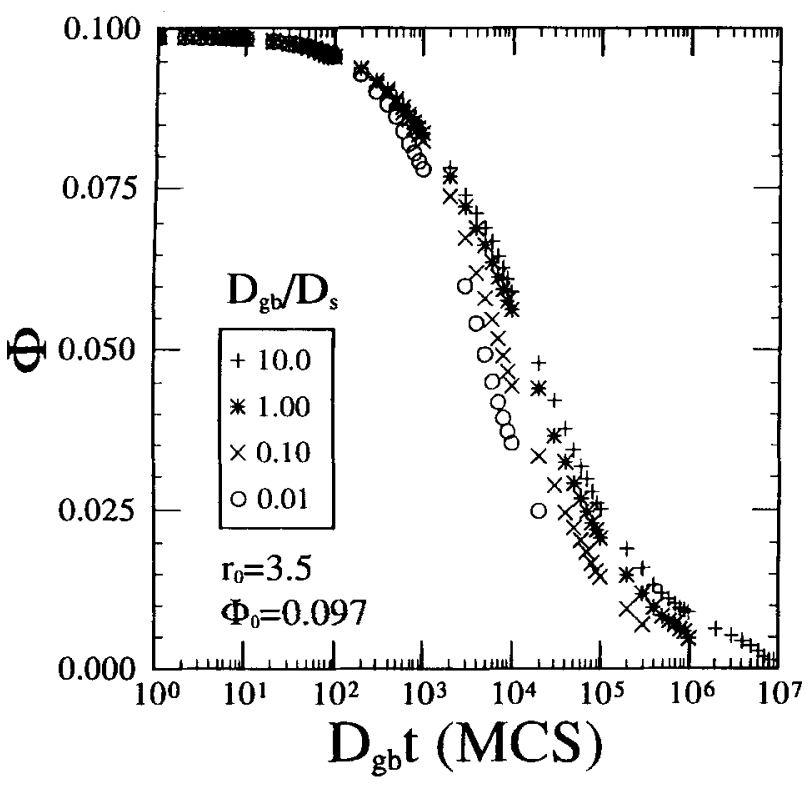

Fig. 9. Dependence of the porosity evolution kinetics on the ratio of $D_{p b} / D_{m}$, where the initial porosity, pore size, and grain size were $9.7 \%, 3.5$, and 15.7 , respectively; time has been normalized by $D_{g b}$.

are presumably related to each other: both the sintering rate and grain growth involve atomic diffusion processes at the grain boundary. In almost all ceramics of practical interest, however, the mobility of a grain boundary is more likely to be dictated by solute drag, as a result of space charge or elastic interactions between impurities and the grain boundary, than by the intrinsic mobility of the grain boundaries.,21 As a result, the mobility of grain boundaries is kinetically tied to lattice diffusion and the overall rate is dependent on the chemistry in the near-grain-boundary region. ${ }^{22}$ Thus, it is more appropriate to regard $D_{g b}$ and $D_{m}$ as two independent quantities, as in our Monte Carlo simulation.

Given the above perspective, we may now associate each of the three kinetic quantities with certain processes of interest in final-stage sintering. $D_{s}$ is associated with the mobility of pores and the ability of pores to remain as equilibrated shapes during their migration and shrinkage, $D_{g b}$ is associated with the shrinkage rate of pores, and $D_{m}$ is associated with grainboundary mobility and grain growth. With the specification of the ratio of surface energy to grain-boundary energy (which determines the dihedral angle) and the assumption that vapor transport and lattice diffusion are unimportant, we believe that our model incorporates all of the basic kinetic and energetic parameters necessary to describe final-stage sintering.

This Monte Carlo simulation technique allows us to understand the relationship between the evolving microstructure and the physical processes that control final-stage sintering. The apparently realistic microstructures generated in our simulation attest to the richness and variety of the features associated with final-stage sintering. Presumably, the kinetic and topological data found in the simulation are influenced by the same variables as are experiments using real materials. Despite this complexity, it is encouraging that the basic scaling relationship of the shrinkage kinetics $\left(\Phi\right.$ vs $\left.D_{g b} t\right)$ is manifested. In part II, ${ }^{11}$ a detailed analysis will be attempted to establish other basic laws governing kinetics and microstructural evolution in such a setting.

Two shortcomings still remain in the correspondence between our model and final-stage sintering in real materials. First, elastic stresses will arise if there is an inhomogeneous depletion of atoms from grain boundaries that fill the pores. In this sense, sintering is an elastodiffusion problem that requires the solution of a set of equations governing stress
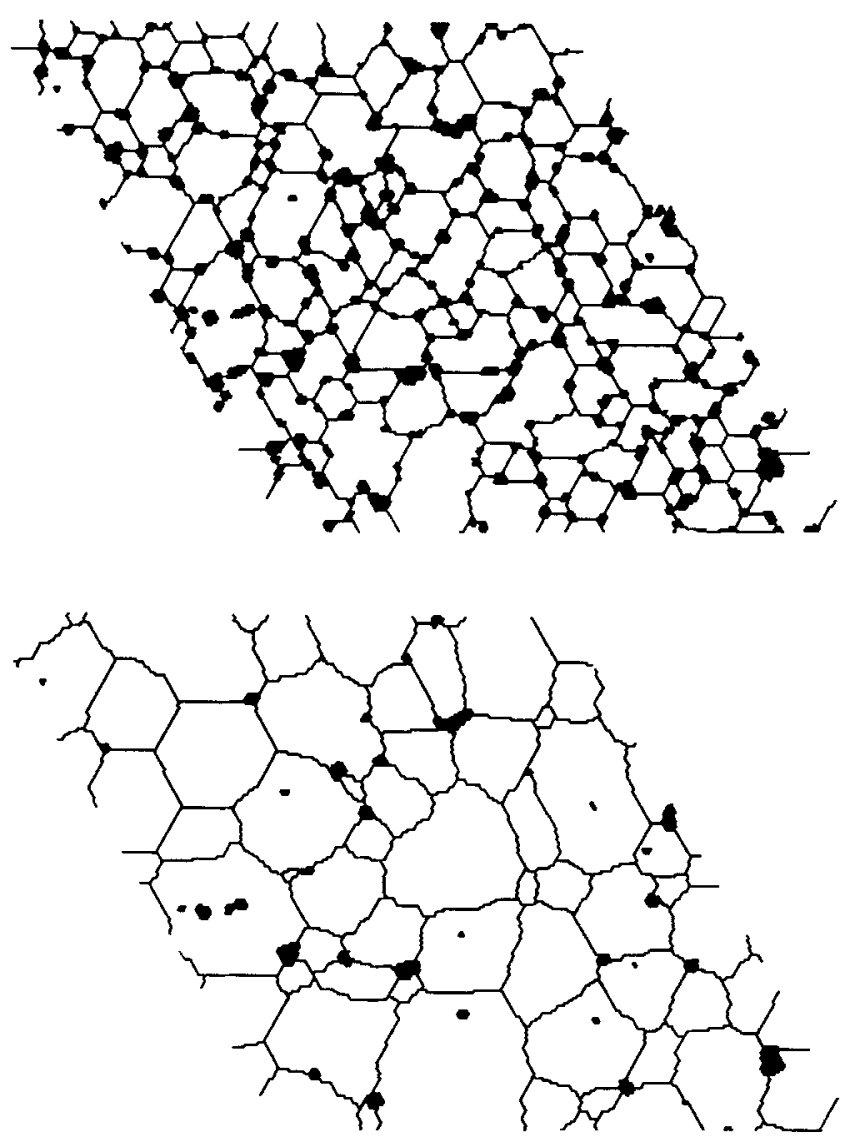

Fig. 10. Microstructural evolution of a porous polycrystalline solid undergoing late-stage sintering, where $D_{g b} / D_{m}$ is 10 and $D_{s} / D_{m}$ is 1 . Initial porosity, pore size, and grain size were $9.8 \%, 3.5$, and 15.7 , respectively.

equilibrium and Fick's law, under appropriate boundary conditions. This is not addressed in our model. Instead, to account for pore shrinkage, we have adopted a simplified method (which is strictly valid only under periodic boundary conditions) and modified it for more general use by requiring that (1) the diffusion distance is related to the distance between a pore and its neighboring pores and (2) that the diffusion takes place along the actual, local grain-boundary network. Because elastic stresses are expected to be largely relaxed when the diffusion distance becomes comparable with the pore spacing, we believe that our model satisfies the major physical constraint that arises from the elasticitydiffusion considerations.

The second deficiency comes from the two-dimensional nature of our model. It will become clear in part II ${ }^{11}$ that some key processes governing the microstructural evolution in a porous polycrystal have different scaling behavior in two and three dimensions. These include the shrinkage equation, the grain growth law, and the breakaway condition for pores. Thus, despite the striking apparent resemblance of the microstructure obtained in our two-dimensional simulations to those observed in real material experiments, an improved computer simulation in three dimensions will still be required to make quantitative predictions of sintering rates in real materials. Nonetheless, this model provides the first direct method of evaluating the role and magnitude of a wide range of physical processes and initial conditions on microstructural evolution during final-stage sintering. Furthermore, because of the availability of relationships between grain growth and coarsening in two and three dimensions, ${ }^{23,24}$ it is, in many cases, possible to extend the understanding developed on the basis of the present two-dimensional simulations to three dimensions. 


\section{Summary}

A computer simulation of final-stage sintering has been performed using a two-dimensional triangular lattice onto which the realistic microstructural features of grains and pores have been mapped. Surface diffusion of vacancies, grain-boundary diffusion for pore shrinkage, and grainboundary migration for grain growth were incorporated into the model based upon Monte Carlo procedures. The dihedral angle was set at $120^{\circ}$ by equating the surface and grainboundary energies. A preliminary study has shown that the simulation procedure accurately reproduces theoretically predicted sintering kinetics under the restricted set of assumptions in which analytical solutions can be obtained.

Over a wide range of diffusivity, initial porosity, and initial pore sizes, the microstructural evolution obtained by the simulation closely resembles microstructures of sintered materials viewed in cross section that have been reported in the literature. Pore shrinkage, grain growth, pore breakaway, and reattachment have all been observed. The porosity decreases monotonically with sintering time and scales with the initial porosity and diffusivity along the grain boundary. Deviations from equilibrium pore shapes under slow surface diffusion or fast grain-boundary diffusion conditions yield slower than expected sintering rates.

\section{APPENDIX A}

Consider the sintering of a periodic, linear array of pores along a grain boundary in two dimensions (see Fig. 3.) These pores are infinitely long cylinders spaced at a distance $\lambda$ apart. The distance between the tips of the pores is $d$, which is smaller than $\lambda$ by the width of the pores. The sintering solution we seek is essentially a variation of the one obtained by Speight and Beere for spherical-cap pores in three dimensions. ${ }^{18}$ As they pointed out for the analogous problem of pore growth under an external tensile stress, pore growth or shrinkage occurs because of the ability of the grain boundary to behave as a perfect source and sink of vacancies. In the steady state, when stress redistribution is complete, vacancy formation or absorption must occur at a constant rate over the entire boundary area. The diffusion equation of vacancies can then be written as

$$
D_{g b} \delta_{g b} \nabla^{2} c=v_{g b}
$$

where $c$ is the vacancy potential normalized by $k T$ (thermal energy), $\delta_{g b}$ is the boundary thickness, $D_{g b}$ is the grainboundary self diffusivity, and $v_{g b}$ is the velocity that the adjacent grains approach each other upon vacancy absorption. To determine $v_{g b}$, we first relate the vacancy potential to the normal tensile stress $\sigma$ through $c=\sigma \Omega / k T$, where $\Omega$ is the atomic volume. The boundary condition at the tip of the pore is $\sigma_{\text {tip }}=\gamma / r$, where $\gamma$ is the surface tension, and $r$ the tip radius of curvature. In the absence of an external stress, the integral of stress over $d$ and the surface tension over the pore must balance to give a zero net stress.

$$
\int_{-\frac{d}{2}}^{\frac{d}{2}} \sigma \mathrm{d} x+\sigma_{\text {tip }}(\lambda-d)=0
$$

Equations (A1) and (A2) and the boundary condition provide the following solution for $c$ :

$$
c=\frac{v_{g b}}{2 D_{g b} \delta_{g b}}\left(x^{2}-\frac{1}{4} d^{2}\right)+\frac{\gamma \Omega}{r k T}
$$

where $x$ is the coordinate originated at the midpoint between two adjacent pores and

$$
v_{g b}=\frac{12 D_{g b} \delta_{g b} \gamma \Omega}{k T}\left(\frac{\lambda}{r d^{3}}\right)
$$

The total shrinkage rate, as pointed out by Speight and Beere, is given by $v_{g b} \lambda$ and not $v_{g b} d$, because the two halves of the pore also approach each other at the same velocity. Thus, if $r<<$, we obtain

$$
\dot{A}=\frac{12 D_{g b} \delta_{g b} \gamma \Omega}{k T}\left(\frac{1}{r \lambda}\right)
$$

where $A$ is the cross-sectional area of the pore and $\dot{A}$ is the rate of change of $A$ in two dimensions. Equation (A5) has the same form as Eq. (3).

Lastly, if the pore has a uniform curvature, i.e., it is fully equilibrated, then

$$
r=(\lambda-d) /[2 \sin (\Psi / 2)]
$$

where $\Psi$ is the dihedral angle. The cross-sectional area of the pore is then proportional to $r^{2}$, given by

$$
A=(\Psi-\sin \Psi) r^{2}
$$

From Eq. (A7) the cubic shrinkage equation for $r$ (Eq. (5)) follows by integration of Eq. (A5).

\section{APPENDIX B}

We here present a model for the characteristic porosity at the onset of final-stage sintering. The model is based on the suggestion of Nichols that the continuous channel of pore space in intermediate-stage sintering eventually breaks down via a Rayleigh instability. ${ }^{25}$

Following Coble, ${ }^{1}$ we model a representative grain in a uniformly packed, partially sintered polycrystal as a tetrakaidecahedron. Tetrakaidecahedra have 14 faces and are connected by 36 edges of equal length $\lambda$. It is well-known that a tetrakaidecahedron is also the Wigner-Seitz cell of a body centered lattice. If we let the cube edge of the body-centered unit cell be $a$, then the tetrakaidecahedron is an octahedron truncated by the cube faces and the octahedral faces are the perpendicular bisectors of eight body diagonals between cube corners and the body center. It can be shown easily that the length of an edge is given by $\lambda=a / 2 \sqrt{2}$. Recall that a Wigner-Seitz cell is always a primitive cell, consisting of one lattice point, and that a cube in a body-centered lattice contains two lattice points, the volume of a tetrakaidecahedron is $a^{3} / 2$, or $8 \sqrt{2} \lambda^{3}$.

Consider an open pore with positive surface tension which is infinitely long, cylindrical in shape, and of radius $r$. Such a cylinder is unstable against longitudinal shape fluctuations of wavelengths in excess of $2 \pi r$ (see Ref. 25 for more details). For a network structure such as the pore channel being discussed here, the boundary condition at the nodes where four pore channels meet (i.e., four grain corners) further requires that the perturbation be a cosine function between two neighboring nodes, with a wavelength $\lambda / n$, where $n$ is an integer. Combining these two conditions, we find that the pore channel is unstable when $\lambda \geq 2 \pi r$.

The critical porosity $\Phi^{*}$ can now be determined. The total pore volume along 36 edges is $36 \pi \lambda r^{2}$ (ignoring corner effects). Only one-third of this volume is contained in a tetrakaidecahedron because edges are shared by three grains. Therefore, the porosity $\Phi^{*}$ is given by $(3 \pi / 2 \sqrt{2})(r / \lambda)^{2}$. At the onset of Rayleigh instability, $\lambda=2 \pi r$, thus, $\Phi^{*}=3 /(8 \sqrt{2 \pi}) \approx 0.0844$. Therefore, final-stage sintering commences when the porosity reaches the critical porosity $\Phi=\Phi^{*} \approx 8.5 \%$. The recent experiment on $\mathrm{Al}_{2} \mathrm{O}_{3}$ supports this prediction. ${ }^{20}$

\section{References}

${ }^{1}$ R. L. Coble, "Sintering Crystalline Solids. I. Intermediate and Final State Diffusion Models and II. Experimental Test of Diffusion Models in Powder Compacts," J. Appl. Phys., 32 [5] 787-99 (1961).

${ }^{2}$ J. E. Burke, "Role of Grain Boundaries in Sintering," J. Am. Ceram. Soc., 40, 80-85 (1957).

${ }^{3}$ R. J. Brook, "Pore-Grain Boundary Interactions and Grain Growth," 
J Am. Ceram. Soc, 52 [1] 56-57 (1969).

${ }^{4}$ C. H. Hsueh, A. G. Evans, and R. L. Coble, "Microstructural Development During Final/Intermediate Stage Sintering. I. Pore/Grain Boundary Separation," Acta Metall., 30, 1269-80 (1982).

${ }^{5}$ M. A. Spears and A.G. Evans, "Microstructure Development During Final/Intermediate Stage Sintering. II. Grain and Pore Coarsening," Acta Metall., 30, 1281-90 (1982).

${ }^{6}$ F. A. Nichols, "Kinetics of Diffusional Motion of Pores in Solids," J. Nucl. Mater, 30, 148 (1969).

7P. G. Shewmon, "Movement of Small Inclusions in Solids by a Tempera ture Gradient," Trans. AIME, 230 [8] 1134-37 (1964).

${ }^{8}$ F. B. Swinkels and M.F. Ashby, "A Second Report on Sintering Dia grams," Acta Metall, 29, 259-81 (1981).

${ }^{9}$ R. J. Brook, "Controlled Grain Growth"; pp. 331-64 in Treatise on Materials Science and Technology, Vol. 9. Edited by F.F.Y. Wang. Academic Press, New York, 1976

${ }^{10}$ M. F. Yan, R. M. Cannon, H. K. Bowen, and U. Chowdhry, "Effect of Grain Size Distribution on Sintered Density," Mater. Sci. Eng., 60, 275-81 (1983).

${ }^{11}$ I-W. Chen, G. N. Hassold, and D. J. Srolovitz, "Computer Simulation of Final Stage Sintering II: Influence of Initial Pore Size," J. Am. Ceram. Soc., $73[10] 2865-72(1990)$

${ }^{12}$ M. P. Anderson, D. J. Srolovitz, G. S. Grest, and P. S. Sahni, "Computer Simulation of Grain Growth I. Kinetics," Acta Metall., 32 [5] 783-91 (1984).

${ }^{13}$ D. J. Srolovitz, M. P. Anderson, P. S. Sahni, and G. S. Grest, "Computer Simulation of Grain Growth II. Grain Size Distribution, Topology, and Local Dynamics," Acta Metall, 32 [5] 793-802 (1984).

${ }^{14}$ D. J. Srolovitz, G. S. Grest, and M.P. Anderson, "Computer Simulation of Recrystallization I. Homogeneous Nucleation and Growth," Acta Metall., 34 [9] 1833-45 (1986).

${ }^{15}$ D. J. Srolovitz, G.S. Grest, M. P. Anderson, and A. D. Rollett, "Com- puter Simulation of Recrystallization II. Heterogeneous Nucleation and Growth," Acta Metall., 36 [8] 2115-28 (1988).

${ }^{16} \mathrm{~J}$. Gayda and D. J. Srolovitz, "A Monte Carlo-Finite Element Model for Strain Energy Controlled Microstructural Evolution: 'Rafting' in Superalloys," Acta Metall., 37 [2] 641-50 (1989).

${ }^{17}$ D. J. Srolovitz and J. F. Scott, "Clock-Model Description of Incommensurate Ferroelectric Films and of Nematic-Liquid-Crystal Films," Phys. Rev. B, 34, 1815-19 (1986).

${ }^{18}$ M.V. Speight and W. Beere, "Vacancy Potential and Void Growth on Grain Boundaries," Met. Sci., 9, 190-91 (1975).

${ }^{19} \mathrm{C}$. A. Handwerker, R. M. Cannon, and R. L. Coble, "Final-Stage Sintering of MgO"; pp. 619-43 in Advances in Ceramics, Vol. 10, Structure and Properties of $\mathrm{MgO}$ and $\mathrm{Al}_{2} \mathrm{O}_{3}$ Ceramics. Edited by W. D. Kingery. American Ceramic Society. Columbus, $\mathrm{OH}, 1984$.

${ }^{20}$ C.P. Cameron and R. Raj, "Grain-Growth Transition During Sintering of Colloidally Prepared Alumina Powder Compacts," J. Am. Ceram. Soc., 71 [12] 1031-35 (1988)

${ }^{21} \mathrm{~S}$. L. Hwang and I-W. Chen, "Grain-Growth Control of Tetragonal Zirconia Polycrystals Using the Space Charge Concept"; to be published in J. Am. Ceram. Soc. (1990).

${ }^{22}$ J.W. Cahn, "Impurity-Drag Effect in Grain Boundary Motion," Acta Metall, 10 [9] 789-98 (1962).

${ }^{23}$ M. P. Anderson G.S. Grest, and D. J. Srolovitz, "Computer Simulation of Normal Grain Growth in Three Dimensions," Philos. Mag. B., 59 [3] 293329 (1989).

${ }^{24}$ M. P. Anderson, G. S. Grest, R. D. Doherty, K. Li, and D. J. Srolovitz, "Inhibition of Grain Growth by Second Phase Particles: Three Dimensional Monte Carlo Computer Simulations," Scr. Metall., 23, 753-58 (1989).

${ }^{25}$ F. A. Nichols and W.W. Mullins, "Surface-(Interface) and VolumeDiffusion Contributions to Morphological Changes Driven by Capillarity," Trans. AIME, 233 [10] 1840-48 (1965). 